\title{
Staged Surgery for Severe Soft Tissue and Bone Loss of the Knee
}

\author{
CK Chan, MBBS, JH Goh, MD, WM Ng, MS ORTH, MK Kwan, MS ORTH, AM Merican, MS ORTH \\ KL Soong, MS ORTH* \\ Department of Orthopaedic Surgery, University Malaya Medical Centre, Kuala Lumpur, Malaysia \\ *Department of Orthopaedic Surgery, Hospital Putrajaya, Putrajaya, Malaysia
}

\begin{abstract}
A 20- year-old female student was involved in a motor vehicle accident. She sustained a severe friction injury to the left knee that resulted in considerable soft tissue and bone loss. There was also damage to the knee extensor mechanism, tibialis anterior muscle, femoral trochlea, the anterior half of the tibial plateau extending distally to the proximal tibia and skin. However, there was no crushing of the limb or resultant neurovascular deficit but cancellous bone and the remainder of the joint were exposed. Repeated surgical debridement was performed and was followed by covering of the soft tissue using a latissimus dorsi free flap and skin grafts. The bony defect was reconstituted with antibiotic bone cement to prevent flap adherence and shrinkage, enhance stability and prevent fracture. The cement was later removed at the time of arthrodesis at which time an ipsilateral double barrel vascularised fibular graft supplemented with autogenously cancellous bone and a ring fixator was used. Computer tomography confirmed union at three months post procedure. The fixator was then removed and a tibialis posterior transfer was performed.
\end{abstract}

Key Words:

Shearing or Friction Injury, Vascularised Fibular Graft, Arthrodesis

\section{INTRODUCTION}

Complex soft tissue defects and bone loss of the knee in the presence of a functional foot pose a treatment dilemma. Case reports and retrospective reviews have been published regarding these defects, but most studies are limited by a small number of patients and/or involve cases related to total knee arthroplasty ${ }^{1,2}$.

We report here a case demonstrating management of a young patient involved in a motor vehicle accident who sustained a complex soft tissue injury with bone loss of the knee. Preserving the limb was the main concern.

\section{CASE REPORT}

A 20-year-old female was involved in a motor vehicle accident in November 2008. She was a passenger in a motorcar when the driver lost control. Most of her body remained in the car except for her left lower limb, which was dragged along the road surface. As a result, she sustained extensive friction burns and degloving injury of the anterior aspect of the left knee involving loss of her distal quadriceps muscle, the patella, the tibialis anterior muscle, the femoral trochlea and the anterior half of the tibial plateau extending distally to the proximal tibia (Figure 1). The remaining joint as well as cancellous bone was exposed but there was no crushing of the limb nor any neurovascular deficit or fractures. The left knee anterior cruciate and medial collateral ligaments were sheared off, however the posterior cruciate and lateral collateral ligaments were intact; the ankle joint was spared, but she was not able to dorsiflex the left ankle due to loss of the tibialis anterior muscle.

The patient was resuscitated in the Emergency Department at the local hospital and underwent surgical debridement on the same day. Systemic and local antibiotics were administered. She was referred to the senior author and was transferred to our institution on the third day after injury. She underwent further wound debridement in preparation for definitive wound covering.

Five days after debridement, a latissimus dorsi free microvascular tissue transfer was performed. The thoracodorsal artery was anastomosed to the superior medial geniculate artery whereas the thoracodorsal vein was anastomosed to the saphenous vein. Vancomycin impregnated cement spacers were shaped and inserted to occupy the femoral and tibial defect. A split skin graft was harvested from the contralateral limb and was applied over the friction burns in the thigh and calf as well as the latissimus dorsi muscle. An external fixator was used to stabilise the knee. The fixator was removed after six weeks at which time the knee was immobilised in a fiberglass cast. Approximately three months later, after numerous consultations with the patient and her mother, a decision was 


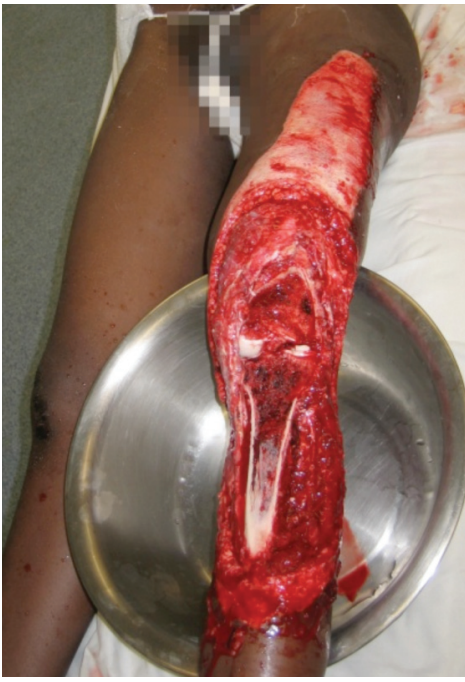

Fig. 1: Shearing injury of the anterior aspect of the left knee. The medullary cavities of femur and tibia as well as the knee joint were exposed. The wound was irrigated with copious amounts of normal saline.

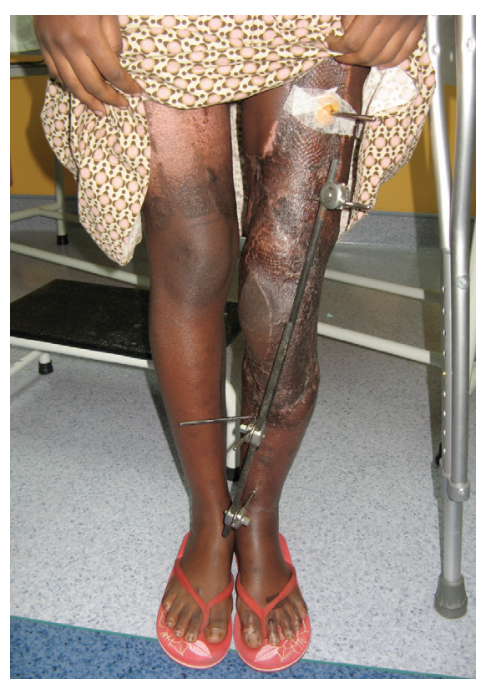

Fig. 3: The patient was able to bear full weight with the external fixator in situ. The latissimus dorsi flap and skin graft has healed.

made to fuse the knee. In view of the bone loss, a combination of external fixation, iliac cancellous bone grafts and pedicled vascularised fibular grafting was chosen to achieve knee arthrodesis. The vancomycin impregnated cement spacer was removed and there were no signs of infection at the time of surgery. Distal femoral and proximal tibial incisions were undertaken with the aid of computer navigation in order to achieve correct mechanical alignment of the limb and a knee flexion angle of 14 degrees. Five degrees of tibiofemoral valgus achieved a mechanical axis which passed through the sagittal centre of the knee.

A twelve centimetre pedicled vascularised fibular graft was harvested from the ipsilateral limb. This was mobilized and
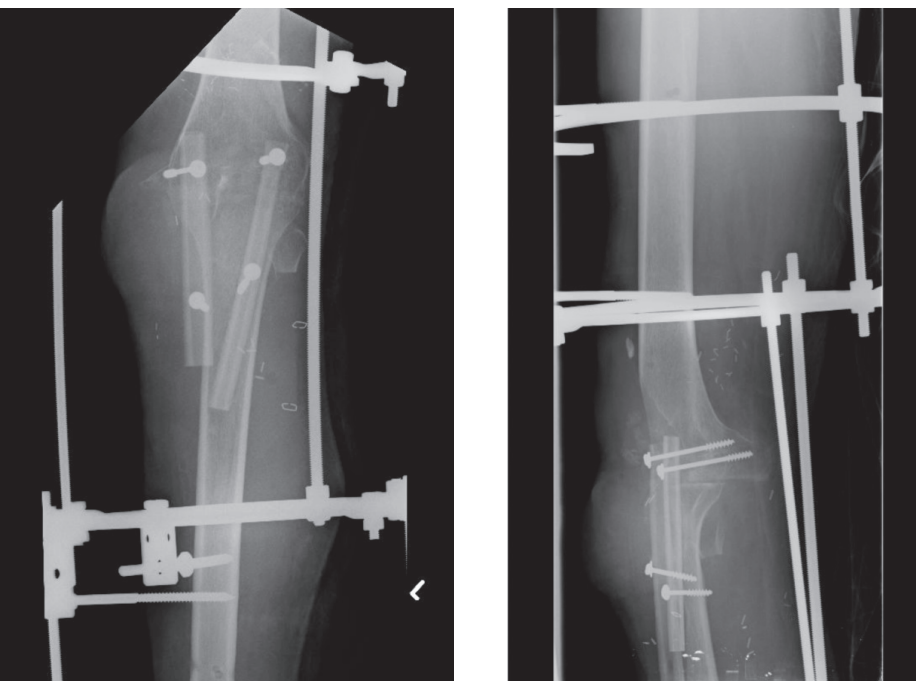

Fig. 2: The pedicled fibular bone was harvested approximately 3 centimetres from the fibular head proximally to 10 centimetres from the distal end of the fibula. The fibular graft was secured with cancellous screws to the host bone. The immediate stability of the knee is enhanced by a ring fixator.

rotated $180^{\circ}$ on its arterial pedicle from the tibio-peroneal trunk. The bone was divided into two halves, delivered proximally to the anterior aspect of the knee and secured in a ' $\mathrm{V}$ ' shape across the knee with screws. This construct was supplemented with cancellous bone graft harvested from the patient's iliac crest. Knee stability was enhanced by application of a ring external fixator (Figure 2).

Doppler ultrasound confirmed vascularity of the pedicled fibular graft. The patient was able to ambulate with partial assistance after the sixth postoperative week. The ring fixator was converted to a uniplanar external fixator at the 10th postoperative week to minimise artefacts during computer tomography (CT). The CT showed successful union of the arthrodesis. The patient was then allowed to fully weight bear (Figure 3). The final surgical procedure was transfer of the tibialis posterior tendon to the tibialis anterior tendon to treat foot drop. During that surgery, the external fixator was removed. The patient adapted well to the fused knee and was satisfied with lower limb function. She subsequently resumed her education at a private college.

\section{DISCUSSION}

Managing patients with complex soft tissue injuries requires a customized approach in order to result in optimal function for the patient and to preserve the lower limb.

The patient described in this report underwent substantial surgical debridement and utilization of a soft tissue flap prior to definitive surgical treatment. Meticulous wound debridement is of utmost importance, as any subsequent procedures will fail if infection and tissue breakdown sets in. Utilization of an antibiotic impregnated cement spacer in 
conjunction with systemic antibiotic therapy in this patient provided efficient local antibiotics delivery, thus allowing for maintenance of ligamentous structures of the knee, enhanced bone quality and prevention of flap adherence and shrinkage ${ }^{3}$. As a result, there was enhanced stability of the knee, prevention of fracture and decreased technical difficulty for subsequent surgical reconstruction. Latissimus dorsi muscle was used as an alternative to extensively used gastronecmius muscles in this patient because the soft tissue deficit was so extensive. Use of latissimus dorsi muscle transfer has a very high success rate (approximately 95\%) ${ }^{4}$. Nevertheless, the pitfall of this procedure lies in the need for microvascular reconstruction facilities that are available mostly in tertiary hospitals. Therefore, early referral to a tertiary hospital with these facilities is essential for limb salvage in this type of injury.

We used a combination method of knee arthrodesis involving a pedicled vascularised fibular graft and external ring fixators to achieve stable knee fusion. Intramedullary nailing and plating was not chosen in the present case due to the extensive bone loss; nailing and plating would have led to insufficient fusion strength and increased risk of infection. Thus, external fixators were the only viable option; however, external fixators alone were not sufficient to provide satisfactory strength for weight bearing and ambulation. The strength issue was addressed by the usage of pedicled vascularised fibular graft, supplemented with ample iliac cancellous bone grafts. Studies by Usui et al. and Rasmussen et al. showed have shown a high union rate of knee arthrodesis with the use of vascularised fibular graft in cases of large bone defect ${ }^{5}$. In this patient, we used pedicled vascularised fibular graft as the donor site due to its' close proximity to the operative site. Careful soft tissue manipulation was essential to avoid vascular injury and related complication of thrombosis and stenosis leading to graft ischemia. Doppler ultrasonography post operation confirmed good arterial flow.
There is no consensus in the literature regarding optimal alignment of knee fusion. Knee flexion at $10-15^{\circ}$ has advantages for allowing an appropriate sitting position and improves gait as compared to setting flexion at $0^{\circ}$. However, there is a resulting limb length discrepancy when knee flexion is set at $15^{\circ}{ }^{6}$. In terms of varus-valgus alignment, 5$7^{\circ}$ valgus is proposed as the ideal alignment to avoid the risk of ipsilateral hip arthritis ${ }^{7}$. Computer navigation was utilized during the procedure to obtain an acceptable lower limb mechanical axis due to the presence of distorted anatomy secondary to the extensive bone loss. The fusion angle of 14 degrees flexion and 5 degrees of valgus is advantageous for achievement of comfortable sitting position and gait. Despite limb length discrepancy of 1.5 centimetres, the patient was satisfied with limb function.

We allowed partial weight bearing at the 6th postoperative week after fusion as we expected sufficient bone healing. External fixators provided additional support, minimizing the risk of fracture or fusion failure. A CT scan performed during the 10th postoperative week confirmed bony union at which the patient was allowed weight-bearing ambulation. The patient was functionally independent, could participate in most social activities and managed to avoid above the knee amputation in this case.

Complex soft tissue injuries of the knee, particularly with bone loss, are challenging to manage. However, staged surgery beginning with meticulous debridement and timely soft tissue coverage, followed by bone reconstruction can avoid complications and preserve the limb. 


\section{REFERENCES}

1. Gerwin M, Rothaus KO, Windsor RE, Brause BD, Insall JN: Gactrocnemius muscle flap coverage of exposed or infected knee prosthesis. Clin Orthop Relat Res 1993; 286: 64-70

2. Greenberg B, LaRossa D, Lotke PA, Murphy JB, Noone RB. Salvage of jeopardized total knee prosthesis: the role of the gactronemius muscle flap. Plast Reconstr Surg 1989; 83: 85-9

3. Booth Jr RE, Lotke PA: The results of spacer block technique in revision of infected total knee arthroplasty. Clin Orthop 1989; 248: 57-60.

4. Frykman GK: Free vascularised flaps for lower extremities reconstruction. Orthopaedics 1986; $39: 505$

5. Usui M, Ishii S, Naito T, Wada T, Nagoya A, Takahashi T, et.al. Arthrodesis of knee joint by vascularized fibular graft. Microsurgery. 1996; 17:2-8.

6. Siller TN, Hadjipavlou A. Knee arthrodesis: long-term results. Can J Surg. 1976; 19: 217-9.

7. Puranen J, Kortelainen P, Jalovaara P. Arthrodesis of the knee with intramedullary nail fixation. J Bone Joint Surg Am. 1990; 72: $433-42$ 